\title{
BMJ Open Risk of ischaemic stroke in patients with migraine: a longitudinal follow-up study using a national sample cohort in South Korea
}

\author{
Sang-Yeon Lee, ${ }^{1}$ Jae-Sung Lim, ${ }^{2}$ Dong Jun Oh, ${ }^{3}$ II Gyu Kong, Hyo Geun Choi ${ }^{4}$
}

To cite: Lee S-Y, Lim J-S, Oh DJ, et al. Risk of ischaemic stroke in patients with migraine: a longitudinal follow-up study using a national sample cohort in South Korea. BMJ Open 2019;9:e027701. doi:10.1136/ bmjopen-2018-027701

- Prepublication history and additional material for this paper are available online. To view these files, please visit the journal online (http://dx.doi. org/10.1136/bmjopen-2018027701).

Received 4 November 2018 Revised 30 January 2019 Accepted 31 January 2019

\section{Check for updates}

(c) Author(s) (or their employer(s)) 2019. Re-use permitted under CC BY-NC. No commercial re-use. See rights and permissions. Published by BMJ.

\section{${ }^{1}$ Department of}

Otorhinolaryngology-Head and Neck Surgery, Seoul National University Hospital, Jongno-gu, The Republic of Korea

${ }^{2}$ Department of Neurology, Hallym University Sacred Heart Hospital, Anyang, The Republic of Korea

${ }^{3}$ Department of Internal Medicine, Asan Medical Center, University of Ulsan College of Medicine, Seoul-si, The Republic of Korea

${ }^{4}$ Department of Laboratory Medicine, HallymUniversity Sacred Heart Hospital, Anyang, The Republic of Korea

Correspondence to

Dr Hyo Geun Choi;

pupen@naver.com

\section{ABSTRACT}

Objective Accumulating evidence has supported the association between migraine and stroke, but the causative association remains unclear. We aimed to investigate the risks of different types of stroke in patients with migraine.

Design A longitudinal follow-up study.

Setting Data collected from a national cohort between 2002 and 2013 by the South Korea Health Insurance Review and Assessment.

Participants We extracted the data from patients with migraine $(n=41585)$ and 1:4 matched controls $(n=166340)$ and analysed the occurrence of ischaemic and haemorrhagic strokes. The migraine group included participants treated for migraine (International Classification of Disease-10 (ICD-10): G43) $\geq 2$ times. Haemorrhagic stroke (I60-I62) and ischaemic stroke (163) were determined based on the admission histories. The crude and adjusted HRs were calculated using Cox proportional hazard models, and the $95 \% \mathrm{Cl}$ were determined. Subgroup analyses stratified by age and sex were also performed.

Results Higher rates of ischaemic stroke were observed in the migraine group $(2.3 \%[964 / 41,585])$ than in the control group (2.0\% [3294/166 340], $\mathrm{P}<0.001)$. The adjusted $\mathrm{HR}$ for ischaemic stroke was $1.18(95 \% \mathrm{Cl}=1.10$ to 1.26$)$ in the migraine group $(\mathrm{P}<0.001)$. Compared with control subjects, participants who reported migraine with aura and migraine without aura had increased adjusted HRs of $1.44(95 \% \mathrm{Cl}=1.09$ to 1.89$)$ and 1.15 $(95 \% \mathrm{Cl}=1.06$ to 1.24$)$, respectively, for ischaemic stroke, but no increased risk of haemorrhagic stroke. In our subgroup analysis, a strong association between migraine and ischaemic stroke was observed in young patients, specifically young women. The contribution of migraine to the occurrence of ischaemic stroke was also observed in middle-aged women and old women (each $\mathrm{P}<0.05$ ).

The risk of haemorrhagic stroke did not reach statistical significance in any age group.

Conclusion Migraine is associated with an increased risk of ischaemic stroke, but not haemorrhagic stroke.

\section{INTRODUCTION}

Migraine is a common neurovascular disorder characterised by recurrent disabling episodes of headache, most often unilateral headache.
Strengths and limitations of this study

The strengths of the present study particularly result from the large cohort based on a complete, national patient sample and the long follow-up period.

- We designed a cohort study to evaluate the risks of different types of stroke in migraineurs.

- Migraine should be considered in assessments of the risk of ischaemic stroke, particularly in young women.

- Confounders linking migraine to stroke, such as cigarette smoking, obesity and prescription information, were not available in the present cohort study based on claim data.

The headache is often accompanied by visual or sensory symptoms, namely, aura. ${ }^{1}$ The annual prevalence of migraine is approximately $8 \%-15 \%$ worldwide, ${ }^{23}$ and in South Korea, the estimated incidence is $6.1 \%{ }^{4}$ Migraine appears to present at a relatively young age $(<45$ years $)$; it is more prevalent in women than men, with a prevalence $>25 \%$ in the 35-39-year-old female population worldwide. ${ }^{1}$ The inclusion of migraine as an independent risk factor for cardiovascular and cerebrovascualr events has been debated for many years. ${ }^{5}$

Stroke is a cerebrovascular condition characterised by limited blood flow to the brain due to a blockage or rupture in the cerebrovascular system. ${ }^{6}$ Stroke is responsible for approximately 700000 deaths annually in the western world. ${ }^{7}$ In South Korea, the estimated stroke incidence is approximately 795000 in people aged $\geq 30$ years. ${ }^{8}$ More than $80 \%$ of stroke cases are ischaemic stroke, and the remaining cases are haemorrhagic stroke; ischaemic and haemorrhagic strokes differ with regard to risk factors, genetic predisposition and mortality rates. ${ }^{910}$

To date, several underlying physiological mechanisms have been suggested to explain 
the association between migraine and stroke. The risk factors for stroke in migraineurs have been widely evaluated, and common comorbidities, including hypertension, obesity, dyslipidaemia and smoking, were validated. ${ }^{11}$ In addition, specific genetic aetiologies associated with vasculopathy can manifest in both patients suffering from migraine and stroke. ${ }^{12}$ A recent investigation employing a mutant mouse model of migraine also indicated that shared genetic risk factors rendered the brain more vulnerable to ischaemic stroke. ${ }^{13}$

Moreover, migraine itself carries an increased risk of ischaemic stroke. Migraine is associated with an increased risk of perioperative ischaemic stroke in a large cohort of surgical patients, suggesting that migraine should be included in the perioperative risk assessment. ${ }^{14}$ Recently, a population-based case-control study in Taiwan reported an association between migraine and ischaemic stroke (adjusted HR: 1.24), (CI: 1.12-1.38). ${ }^{3}$ Similarly, an increased risk of ischaemic stroke has been consistently observed in migraineurs, especially in those diagnosed with migraine with aura. ${ }^{3}$ 15-18 Furthermore, accumulating evidence has supported the association between migraine and haemorrhagic stroke,${ }^{19}{ }^{20}$ but the causative association currently remains unclear.

Given the differences in the natures of haemorrhagic stroke and ischaemic stroke in patients, including risk factors and genetic predispositions, ${ }^{9}{ }^{10}$ we hypothesised that the contributions of migraine to an increased risk of stroke differ according to the stroke type. Thus, stroke types should be analysed separately. Additionally, stroke is a leading cause of mortality; therefore, validation of the association between migraine and stroke may facilitate treatment and prognosis in these cases. Here, using a national South Korea population-based sample cohort, we examined the associations between migraine and different types of stroke.

\section{MATERIALS AND METHODS}

\section{Study population and data collection}

This national cohort study used data from the South Korea National Health Insurance Service-National Sample Cohort (NHIS-NSC). The South Korea NHIS selects samples directly from the entire population database to prevent non-sampling errors. Approximately $2 \%$ of the samples (one million) were selected from the entire South Korea population (50 million). The selected data were classified into 1476 levels (age [18 categories], sex [two categories] and income level [41 categories]) using randomised, stratified, systematic sampling methods via proportional allocation to represent the entire population. A previous study verified the appropriateness of the sample after data selection. ${ }^{21}$ The National Health Insurance Sharing Service provides a detailed description of the methods used to perform these procedures. ${ }^{22}$ This cohort database included (i) personal information, (ii) health insurance claim codes (procedures and prescriptions), (iii) diagnostic codes based on the
International Classification of Disease-10 (ICD-10), (iv) death records from the South Korea National Statistical Office (using the South Korea Standard Classification of disease), (v) socioeconomic data (residence and income) and (vi) medical examination data for each participant from 2002 to 2013.

All South Korea citizens are recognised by a 13-digit resident registration number from birth to death. Therefore, exact population statistics have been determined using this database. All South Korea are required to enrol in the NHIS. All South Korea hospitals and clinics use the 13-digit resident registration number to register individual patients in the medical insurance system. Therefore, the risk of overlapping medical records is minimal, even if a patient moves from one place to another. All medical treatments in South Korea are tracked without exception using the Health Insurance Review \& Assessment (HIRA) system. In South Korea, the law states that a notice of death must be provided to an administrative entity before a funeralcan be held. Medical doctors record the date and cause of death on a death certificate.

\section{Patients and public involvement}

No patients or public were involved in the present study.

\section{Participant selection}

Of the 1125691 patients with 114369638 medical claim codes, participants diagnosed with migraine (ICD-10: G43) were included. Among these participants, those who were treated $\geq$ two times $(n=45587)$ were selected. The participants were followed for 12 years.

The history of admission for haemorrhagic stroke (I60: subarachnoid haemorrhage, I61: intracerebral haemorrhage and I62: other non-traumatic intracranial haemorrhage) and ischaemic stroke (I63: cerebral infarction) was identified using ICD-10 codes. We selected the participants who were treated $\geq$ one time. These methods were used in other studies evaluating the incidence of stoke in South Korea. ${ }^{8} 23$

The participants with migraine were matched 1:4 with participants (control group) who had never been diagnosed with a migraine from 2002 through 2013 in this cohort. The control group was selected from the total population $(\mathrm{n}=1080104)$. The matches were processed for age, group, sex, income group, region of residence and medical histories (age, sex, income, region of residence, hypertension, diabetes and dyslipidaemia histories). We sorted the participants in the control group using a random number order and then selected them from top to bottom to prevent selection bias when selecting the matched control participants. We assumed that the matched control participants were enrolled at the same time as each matched participant with migraine (index date). Therefore, control group members who died before the index date were excluded. In both the migraine and control groups, participants with histories of haemorrhagic or ischaemic stroke before the index date were excluded. In the migraine group, 438 participants 


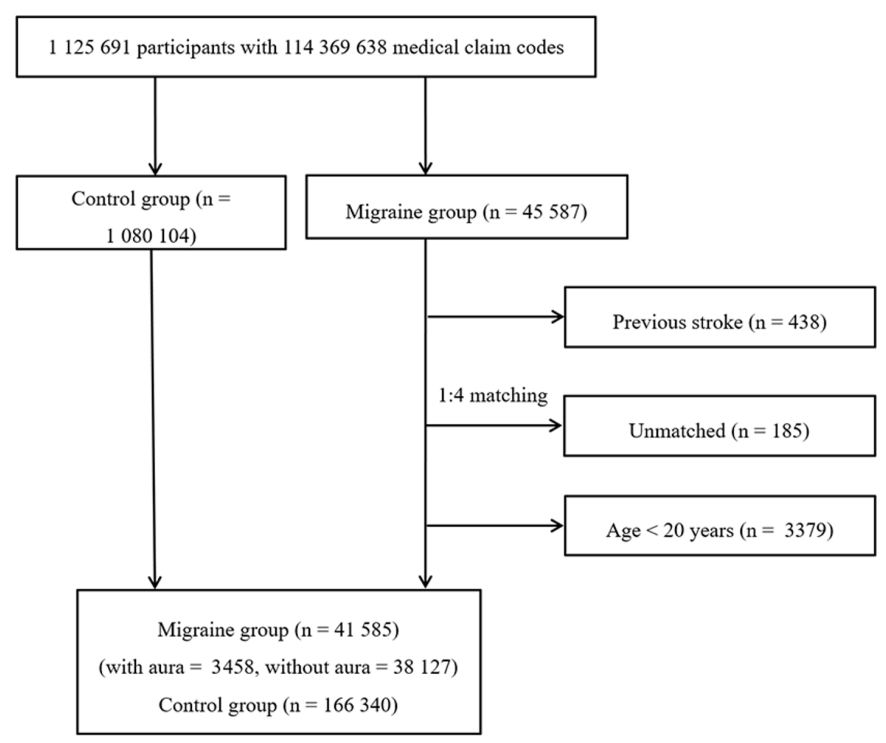

Figure 1 A schematic illustrating the participant selection process used in the present study. Of a total of 1125691 participants, 41585 participants with migraine were matched with 166340 control participants for age, group, sex, income level, region of residence and medical histories.

were excluded. The participants with migraine for whom we were unable to identify sufficient numbers of matching participants were excluded $(\mathrm{n}=185)$. We excluded participants aged less than 20 years $(n=3379)$. Finally, 1:4 matching resulted in the inclusion of 41585 participants with migraine (migraine with aura $=3458$, migraine without aura $=38127)$ and 166340 control participants (figure 1).

\section{Variables}

The age groups were classified using 5-year intervals: aged $20-24,25-29,30-34 \ldots$, and $85+$ years. Fourteen age groups were designated. The income groups were initially divided into 41 classes (one health aid class, 20 self-employment health insurance classes and 20 employment health insurance classes). These groups were recategorised into 11 classes (class 1 [lowest income]-class 11 [highest income]). The region of residence was divided into 16 areas according to administrative district. These regions were regrouped into urban (Seoul, Busan, Daegu, Incheon, Gwangju, Daejeon and Ulsan) and rural (Gyeonggi, Gangwon, Chungcheongbuk, Chungcheongnam, Jeollabuk, Jeollanam, Gyeongsangbuk, Gyeongsangnam and Jeju) areas. The medical histories of the participants were evaluated using ICD-10 codes. For an accurate diagnosis, hypertension (I10 and I15), diabetes (E10-E14) and dyslipidaemia (E78) were recorded if the participants were treated $\geq$ two times. Using the Charlson comorbidity index, we selected some of confounders that might affect the association between migraine and stroke as confounders based on literature review. We performed the analysis with added variables, including congestive heart failure, ${ }^{24}$ myocardial infarction, ${ }^{25}$ peripheral vascular disease,$^{26}$ pulmonary disease, ${ }^{27}$ liver disease $^{28}$ and depression histories. ${ }^{29}{ }^{30}$ Thus, the new model was adjusted for age, sex, income, region of residence, hypertension, diabetes, dyslipidaemia, congestive heart failure, myocardial infarction, peripheral vascular disease, pulmonary disease, liver disease and depression histories.

\section{Statistical analyses}

$\chi^{2}$ tests were used to compare the general characteristics between the migraine and control groups. For the analysis of the HRs of migraine for haemorrhagic stroke and ischaemic stroke, Cox proportional hazard models were used, and 95\% CIs were calculated. In these analyses, crude (simple) and adjusted (age, sex, income, region of residence, hypertension, diabetes, dyslipidaemia, congestive heart failure, myocardial infarction, peripheral vascular disease, pulmonary disease, liver disease and depression histories) models were used. For the subgroup analysis, we stratified the participants by age and sex (aged 20-39 years, 40-59 years, 60+ years; men and women). Two-tailed analyses were conducted, and $p$ values less than 0.05 were considered significant. The results were statistically analysed using SPSS V.21.0 software (IBM, Armonk, NY, USA).

\section{RESULTS}

The mean follow-up period was $80.9(\mathrm{SD}=41.6)$ months for patients with migraine and $80.9(\mathrm{SD}=41.6)$ months for controls. The rates of haemorrhagic stroke were similar in the migraine group $(0.7 \%$ [295/41 585]) and the control group (0.7\% [1113/166 340], $\mathrm{P}=0.370$, table 1$)$. Higher rates of ischaemic stroke were observed in the migraine group (2.3\% [964/41 585]) than in the control group (2.0\% [3294/166 340], $\mathrm{P}<0.001)$. The general characteristics (age, sex, income, region of residence, hypertension, diabetes and dyslipidaemia histories) of participants were the same due to the matching protocol $(\mathrm{P}=1.000)$.

In the subgroup analyses, none of the crude and adjusted HRs for haemorrhagic stroke reached statistical significance (table 3). Among the patients with ischaemic stroke, young women, middle-aged women and old women showed statistically significant differences $($ each $\mathrm{P}<0.05)$. The significant adjusted HRs were 2.31 (95\% CI=1.39 to 3.82$)$ in young women (aged $20-39$ years); $1.32(95 \% \mathrm{CI}=1.08$ to 1.61$)$ in middle-aged women (aged $40-59$ years); and 1.18 $(95 \% \mathrm{CI}=1.06$ to 1.30$)$ in $\geq 60$ year-old women. Moreover, descriptive statistical analyses of the migraine prevalence and ischaemic stroke occurrence within subgroups revealed statistically significant differences in young men, young women, middle-aged women, old men and old women. On the other hand, the risk of haemorrhagic stroke in patients with migraine was not significantly different from the control group (online supplementary table 1 ). 
Table 1 General characteristics of the participants

\begin{tabular}{|c|c|c|c|}
\hline \multirow[b]{2}{*}{ Characteristics } & \multicolumn{3}{|c|}{ Total participants } \\
\hline & $\begin{array}{l}\text { Migraine (n, } \\
\%)\end{array}$ & Control (n, \%) & $P$ value \\
\hline Age (years) & & & 1.000 \\
\hline 20-24 & $1994(4.8)$ & $7976(4.8)$ & \\
\hline 25-29 & $2649(6.4)$ & $10596(6.4)$ & \\
\hline 30-34 & $3640(8.8)$ & $14560(8.8)$ & \\
\hline 35-39 & 4309 (10.4) & $17236(10.4)$ & \\
\hline $40-44$ & 4859 (11.7) & 19436 (11.7) & \\
\hline $45-49$ & $5187(12.5)$ & $20748(12.5)$ & \\
\hline $50-54$ & $4512(10.9)$ & 18048 (10.9) & \\
\hline $55-59$ & $3508(8.4)$ & $14032(8.4)$ & \\
\hline $60-64$ & $3209(7.7)$ & $12836(7.7)$ & \\
\hline $65-69$ & $3049(7.3)$ & $12196(7.3)$ & \\
\hline $70-74$ & $2328(5.6)$ & $9312(5.6)$ & \\
\hline $75-79$ & $1423(3.4)$ & $5692(3.4)$ & \\
\hline $80-84$ & 651 (1.6) & $2604(1.6)$ & \\
\hline $85+$ & $267(0.6)$ & $1068(0.6)$ & \\
\hline Sex & & & 1.000 \\
\hline Male & $10476(25.2)$ & 41904 (25.2) & \\
\hline Female & $31109(74.8)$ & $124436(74.8)$ & \\
\hline Income & & & 1.000 \\
\hline 1 (lowest) & 837 (2.0) & $3348(2.0)$ & \\
\hline 2 & $3170(7.6)$ & $12680(7.6)$ & \\
\hline 3 & $3004(7.2)$ & $12016(7.2)$ & \\
\hline 4 & $3131(7.5)$ & $12524(7.5)$ & \\
\hline 5 & $3345(8.0)$ & $13380(8.0)$ & \\
\hline 6 & $3615(8.7)$ & $14460(8.7)$ & \\
\hline 7 & 3908 (9.4) & $15632(9.4)$ & \\
\hline 8 & $4411(10.6)$ & $17644(10.6)$ & \\
\hline 9 & $4908(11.8)$ & $19632(11.8)$ & \\
\hline 10 & $5456(13.1)$ & $21824(13.1)$ & \\
\hline 11 (highest) & $5800(13.9)$ & $23200(13.9)$ & \\
\hline \multicolumn{2}{|c|}{ Region of residence } & & 1.000 \\
\hline Urban & $17959(43.2)$ & $71836(43.2)$ & \\
\hline Rural & $23626(56.8)$ & $94504(56.8)$ & \\
\hline Hypertension & 16209 (39.0) & $64836(39.0)$ & 1.000 \\
\hline Diabetes & $7261(17.5)$ & 29044 (17.5) & 1.000 \\
\hline Dyslipidaemia & 12837 (30.9) & 51348 (30.9) & 1.000 \\
\hline $\begin{array}{l}\text { Congestive heart } \\
\text { failure }\end{array}$ & $2030(4.9)$ & $6761(4.1)$ & $<0.001^{*}$ \\
\hline $\begin{array}{l}\text { Myocardial } \\
\text { infarction }\end{array}$ & $913(2.2)$ & $3330(2.0)$ & $0.013^{*}$ \\
\hline $\begin{array}{l}\text { Peripheral } \\
\text { vascular disease }\end{array}$ & 7942 (19.1) & 20217 (12.2) & $<0.001^{*}$ \\
\hline $\begin{array}{l}\text { Pulmonary } \\
\text { disease }\end{array}$ & $29540(71.0)$ & 94811 (57.0) & $<0.001^{*}$ \\
\hline
\end{tabular}

Continued
Table 1 Continued

\begin{tabular}{|c|c|c|c|}
\hline \multirow[b]{2}{*}{ Characteristics } & \multicolumn{3}{|c|}{ Total participants } \\
\hline & $\begin{array}{l}\text { Migraine (n, } \\
\%)\end{array}$ & Control (n, \%) & $P$ value \\
\hline Liver disease & 5087 (12.2) & 15317 (9.2) & $<0.001^{*}$ \\
\hline Depression & 7808 (18.8) & 15269 (9.2) & $<0.001^{*}$ \\
\hline $\begin{array}{l}\text { Haemorrhagic } \\
\text { stroke }\end{array}$ & $295(0.7)$ & $1113(0.7)$ & 0.370 \\
\hline Ischaemic stroke & 964 (2.3) & 3294 (2.0) & $<0.001^{*}$ \\
\hline
\end{tabular}

The crude and adjusted HRs for haemorrhagic stroke were 1.06 $(95 \% \mathrm{Cl}=0.93$ to $1.21, \mathrm{P}=0.369)$ and $1.10(95 \% \mathrm{Cl}=0.96$ to 1.25 , $\mathrm{P}=0.172$ ), respectively, in the migraine group (table 2). The crude and adjusted HRs for ischaemic stroke were 1.17 (95\% $\mathrm{Cl}=1.09$ to 1.26$)$ and $1.18(95 \% \mathrm{Cl}=1.08$ to 1.25$)$, respectively, in the migraine group (each $\mathrm{P}<0.001$ ).

${ }^{*} \chi^{2}$ test. Differences were considered significant at $P<0.05$.

Compared with the control group, participants who reported migraine with aura and migraine without aura had increased adjusted HRs of 1.44 (95\% CI=1.09 to 1.89) and 1.15 (95\% CI=1.06 to 1.24) for ischaemic stroke, respectively, but no increased risk of haemorrhagic stroke (table 4).

\section{DISCUSSION}

Migraine appeared to increase the risk of ischaemic stroke, but not haemorrhagic stroke. The risk of ischaemic stroke was 1.18-fold greater in the patients with migraine than in the matched control subjects, even after adjustment for confounding factors. In the subgroup analyses stratified by age and sex, significant correlations with ischaemic stroke were primarily observed in young women, middle-aged women and old women. In contrast, the risk of haemorrhagic stroke was similar in patients with migraine and the matched control patients.

Migraine, particularly migraine with aura, is an established risk factor for ischaemic stroke. In this study, compared with the control group, participants who reported migraine with aura had an increased adjusted HR of 1.44 (95\% $\mathrm{CI}=1.09$ to 1.89 ) for ischaemic stroke. Migraine with aura particularly increases the risk of ischaemic stroke, based on the results of large population-based cohort studies and meta-analyses. ${ }^{3}{ }^{15-18}$ Cortical spreading depression (CSD) is a self-propagating wave of depolarization across the cerebral cortex at a slow velocity of $3-5 \mathrm{~mm} / \mathrm{min} .{ }^{31} \mathrm{CSD}$ has been implicated in migraine with aura, which plays a critical role in the pathophysiology of ischaemic stroke. In an experimental study, CSD altered the tone of resistance vessels, causing hypoperfusion in tissues at risk for progressive damage, namely, cortical spreading ischaemia. ${ }^{32}$ In addition, the inflammatory cascade of the neurovascular system, which is characterised by endothelial dysfunction and coagulation abnormalities, may contribute to the development of ischaemic stroke. In the aura phase, the endothelium activates coagulation and thrombosis, which 
Table 2 Crude and adjusted HRs (95\% Cl) of migraine for haemorrhagic stroke and ischaemic stroke

\begin{tabular}{|c|c|c|c|c|c|c|c|c|}
\hline \multirow[b]{2}{*}{ Characteristics } & \multicolumn{4}{|c|}{ Haemorrhagic stroke } & \multicolumn{4}{|l|}{ Ischaemic stroke } \\
\hline & Crude & $P$ values & Adjusted* & $P$ values & Crude & P values & Adjusted* & $P$ values \\
\hline Migraine & & 0.369 & & 0.172 & & $<0.001 \dagger$ & & $<0.001 \dagger$ \\
\hline No & 1.00 & & 1.00 & & 1.00 & & 1.00 & \\
\hline
\end{tabular}

*Model adjusted for age, sex, income, region of residence, hypertension, diabetes, dyslipidaemia, congestive heart failure, myocardial infarction, peripheral vascular disease, pulmonary disease, liver disease and depression histories.

†Cox proportional hazard regression model; differences were considered significant at $\mathrm{P}<0.05$.

are mediated by inflammatory cytokines and endothelial biomarkers. ${ }^{334}$ Moreover, specific genetic aetiologies, such as factor V Leiden G 619 1A, prothrombin G20210A and familial hemiplegic migraine type 1 , have been reported in patients diagnosed with migraine with aura. These shared genetic factors may precipitate the susceptibility to CSD, which may explain the association between migraine and stroke. $^{35}$

A growing body of evidence regarding the increased risk of ischaemic stroke in patients with migraine without aura. Consistent with the findings from patients reporting migraine with aura, participants who reported migraine without aura had an increased adjusted HR of $1.15(95 \%$ $\mathrm{CI}=1.06$ to 1.24 ) for ischaemic stroke compared with the control group. Notably, a significant association was observed in cases of ischaemic stroke resulting from cervical artery dissection. ${ }^{36}$ In addition, genetic overlaps among subjects with migraine without aura, large artery stroke, and cardioembolic stroke have been identified. ${ }^{37}$ However, strong evidence has been not reported. A recent large population-based study reported an statistically insignificant risk of ischaemic stroke in patients with

Table 3 Subgroup analysis of crude and adjusted HRs (95\% Cl) of migraine for haemorrhagic stroke and ischaemic stroke

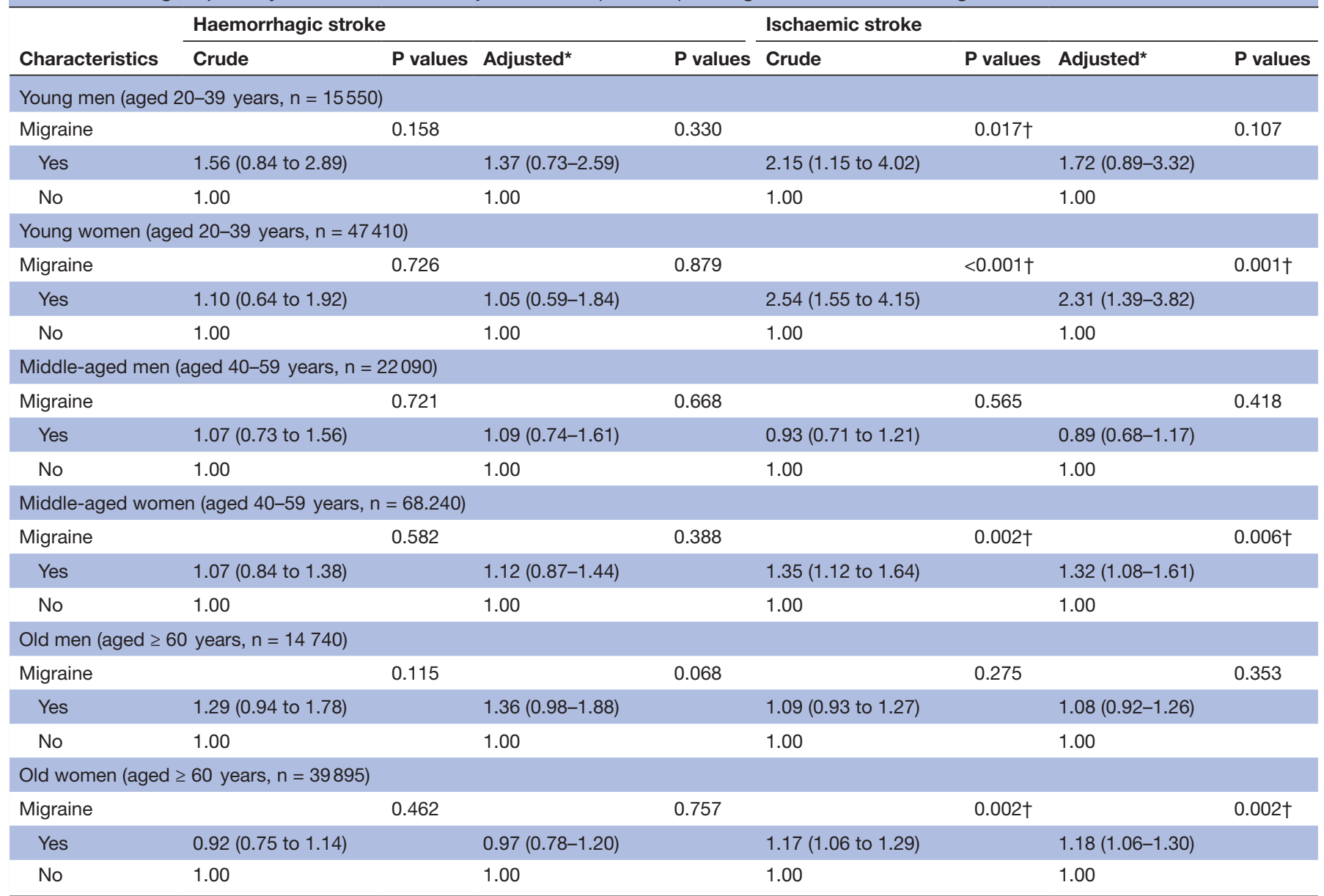

*Model adjusted for age, sex, income, region of residence, hypertension, diabetes, dyslipidaemia, congestive heart failure, myocardial infarction, peripheral vascular disease, pulmonary disease, liver disease, and depression histories.

†Cox proportional hazard regression model; differences were considered significant at $\mathrm{P}<0.05$. 
Table 4 Subgroup analysis of crude and adjusted HRs $(95 \% \mathrm{Cl})$ of migraine for haemorrhagic stroke and ischaemic stroke

\begin{tabular}{|c|c|c|c|c|c|c|c|c|}
\hline \multirow[b]{2}{*}{ Characteristics } & \multicolumn{4}{|c|}{ Haemorrhagic stroke } & \multicolumn{4}{|l|}{ Ischaemic stroke } \\
\hline & Crude & $P$ values & Adjusted* & $P$ values & Crude & $P$ values & Adjusted $^{*}$ & $P$ values \\
\hline \multicolumn{9}{|c|}{ Migraine with aura $(n=17290)$} \\
\hline Yes & $1.17(0.72$ to 1.90$)$ & & $1.19(0.72-1.96)$ & & 1.45 (1.11 to 1.88$)$ & & $1.44(1.09-1.89)$ & \\
\hline No & 1.00 & & 1.00 & & 1.00 & & 1.00 & \\
\hline Yes & 1.05 (0.92 to 1.20$)$ & & $1.09(0.95-1.25)$ & & $1.16(1.07$ to 1.24$)$ & & $1.15(1.06-1.24)$ & \\
\hline No & 1.00 & & 1.00 & & 1.00 & & 1.00 & \\
\hline
\end{tabular}

*Model adjusted for age, sex, income, region of residence, hypertension, diabetes, dyslipidaemia, congestive heart failure, myocardial infarction, peripheral vascular disease, pulmonary disease, liver disease and depression histories.

†Cox proportional hazard regression model; differences were considered significant at $\mathrm{P}<0.05$.

migraine without aura but an increased risk in patients with migraine with aura. ${ }^{3}$ The study suggested that the difference may be due to changes in the lipid profile, the susceptibility to thrombosis, and genetic predisposition. ${ }^{3}$ A meta-analysis did not identify a significant association between migraine without aura and ischaemic stroke. ${ }^{18}$ In the present study, we did not differentiate between migraine subtypes, such as migraine with and without aura, when analysing the risk of stroke. Therefore, the risk of ischaemic stroke in participants with migraine was relatively low but statistically significant compared with other studies investigating specific conditions.

Notably, in the present study, migraine was not associated with an increased risk of haemorrhagic stroke. In contrast to the findings from the present study, a recent meta-analysis based on four case-control and four cohort studies concluded that migraine may increase the risk of haemorrhagic stroke. ${ }^{20}$ However, a recent large population-based case-control study did not show an association with different types of haemorrhagic stroke, including intracerebral haemorrhage and subarachnoid haemorrhage ${ }^{38}$ Migraine was an independent risk factor for aneurismal rupture $(\mathrm{OR}(\mathrm{OR})=2.4 ; 95 \% \mathrm{CI} 1.1$ to 5.1$)$ in a case-control study, ${ }^{39}$ and even if headache is a premonitory symptom of aneurismal rupture, recall biases might have affected the results of the study. Overall, the relatively small number of haemorrhagic stroke cases might have resulted in decreased statistical power.

In our subgroup analysis, a strong correlation between migraine and ischaemic stroke was observed in young patients, specifically young women (aged 20-39 years). Based on a consensus statement from the European Headache Federation, ${ }^{40}$ the use of a combined hormonal contraceptive may further increase the risk of ischaemic stroke in patients with migraine, specifically migraine with aura, supporting our results. Because the predisposing factors for stroke increase the incidence of ischaemic stroke with age, ${ }^{41}$ migraine itself rather than other risk factors might be implicated in the increased risk of ischaemic stroke. In contrast, a recent large, population-based cohort study in Taiwan with a relatively short follow-up duration of 3.6 years did not show a significant association in the subgroups of women and patients aged $<45$ years. ${ }^{3}$ This discrepancy might be due to differences in the study design, follow-up duration and ethnic composition of the study population. Recently, a similar trend for this association was also reported, ${ }^{3}$ but it requires further confirmation due to the lack of evidence at present.

The present study has several strengths. The results of this study are consistent with the findings of our previous studies using the HIRA-NSC. ${ }^{42-44}$ We examined a very large, representative and nationwide population. Because the NHIS data cover all citizens of South Korea without exception, no participants were lost during follow-up. The control group was randomly selected and matched based on age, sex, income, region of residence and medical history to decrease any confounding effects. An adjusted hazard model was used to further minimise the impacts of confounders. Because migraine attacks might be frequently repeated, we opted to include patients with migraine who underwent treatment at least twice based on the ICD-10 code to increase the validity of the study (G43). In a previous study, the prevalence of migraine in South Korea was reported to be approximately $6.1 \% .^{4}$ This value appeared to be slightly higher than the value reported in the present study, which was $3.7 \%$ (41 585/1 125691$)$, based on strict inclusion criteria for migraine. In addition, the patients with migraine were followed for 12 years in the present study. Long study periods enable the recruitment of a large study population and allow researchers to observe and analyse the delayed effects of migraine on stroke.

However, the present study has certain limitations that should be addressed in future studies. First, we were unable to extract information on stroke subtypes and location from the data used in the present study. The impact of migraine on each type of pathophysiology might vary when considering different functional outcomes, survival and recurrence according to stroke subtype. ${ }^{38}{ }^{45}$ However, in the present study, the distribution of stroke subtypes was not available. The classification of stroke type can lead to 
a significantly decreased incidence of each disease. As an insufficient number of cases cause inappropriate comparisons due to lower statistical power, the inclusion of the distribution of stroke subtypes would hamper our ability to draw a clear conclusion from the available data. In particular, the absolute incidence of haemorrhagic stroke is very low in general practice; therefore, most of studies did not provide information about subtypes, such as the presence of an aneurysm, although the association is positive in public health studies. ${ }^{38}$ Second, a firm correlation between the frequency of migraine attacks and the risk of stroke has been reported, ${ }^{46}$ but we were unable to confirm this correlation using the claim data analysed in the present study. Similarly, the duration and severity of migraine were inconsistent among the study population. Patients with migraine who had mild symptoms may not have received a consultation at the clinic; therefore, the impact of migraine on stroke may be underestimated in this study. Third, although this study attempted to include a large, representative patient population and subsequently matched and adjusted for possible confounders, the risk factors for stroke in patients with migraine, such as cigarette smoking, obesity and migraine-specific drugs, were not available in the insurance database. ${ }^{1146}$ Finally, in the present study, the incidence of migraine was 3.7\% (41 585 of 1125691 participants) after employing a strict matching protocol. Before matching, the incidence of migraine in South Korea was 4.1\% (45 587 of 1125691 participants). This value is consistent with previous cohort study in South Korea, indicating that the overall prevalence rate of migraine was $6.1 \%,{ }^{4}$ despite the use of different study designs. Given the global prevalence of migraine of approximately $8 \%-15 \%,{ }^{23}$ the incidence of migraine in South Korea is relatively low because of limited claim data. Only patients with migraine who visited the hospital are recorded. In other words, data are not available for patients with weak migraine symptoms or patients who do not visit a hospital, leading to a lower incidence. Based on a population-based epidemiological study of migraine in South Korea, only $24.4 \%$ of patients ever consulted a doctor for headache and only $3.3 \%$ of patients were prescribed a drug by a doctor. ${ }^{47}$

\section{CONCLUSIONS}

Based on a large population-based cohort study in South Korea, migraine increased the risk of ischaemic stroke, but not haemorrhagic stroke. The subgroup of patients at the highest risk of developing ischaemic stroke was young women.

Acknowledgements We thank the participants and examiners of Korean Health Insurance Review and Assessment for participating in this cohort study and providing the data, respectively.

Contributors As the first author, S-YL wrote the manuscript. DJO and J-SL analysed and interpreted the data. IGK processed the data. HGC conceptualised, wrote and reviewed the manuscript.

Funding This work was supported by the Hallym University Research Fund and in part by a research grant (NRF-2015-R1D1A1A01060860) from the National Research Foundation (NRF) of Korea.
Competing interests None declared.

Patient consent for publication Not required.

Ethics approval The ethics committee of Hallym University (2014-I148) approved the use of these data.

Provenance and peer review Not commissioned; externally peer reviewed.

Data sharing statement Data from the Korean National Health Insurance ServiceNational Sample Cohort (NHIS-NSC) in Korea.

Open access This is an open access article distributed in accordance with the Creative Commons Attribution Non Commercial (CC BY-NC 4.0) license, which permits others to distribute, remix, adapt, build upon this work non-commercially, and license their derivative works on different terms, provided the original work is properly cited, appropriate credit is given, any changes made indicated, and the use is non-commercial. See: http://creativecommons.org/licenses/by-nc/4.0/.

\section{REFERENCES}

1. Charles A. Migraine. N Engl J Med 2017;377:553-61.

2. Lipton RB, Scher Al, Kolodner K, et al. Migraine in the United States: epidemiology and patterns of health care use. Neurology 2002;58:885-94.

3. Peng KP, Chen YT, Fuh JL, et al. Migraine and incidence of ischemic stroke: a nationwide population-based study. Cephalalgia 2017;37:327-35.

4. Kim BK, Chu MK, Lee TG, et al. Prevalence and impact of migraine and tension-type headache in Korea. J Clin Neurol 2012;8:204-11.

5. Wood H. Migraine: Migraine - a risk factor for cardiovascular disease? Nat Rev Neurol 2018;14:194-5.

6. Feigin VL, Lawes CM, Bennett DA, et al. Worldwide stroke incidence and early case fatality reported in 56 population-based studies: a systematic review. Lancet Neurol 2009;8:355-69.

7. Ojaghihaghighi S, Vahdati SS, Mikaeilpour A, et al. Comparison of neurological clinical manifestation in patients with hemorrhagic and ischemic stroke. World J Emerg Med 2017;8:34.

8. Hong KS, Bang OY, Kang DW, et al. Stroke statistics in Korea: part I. Epidemiology and risk factors: a report from the korean stroke society and clinical research center for stroke. J Stroke 2013;15:2-20.

9. Andersen KK, Olsen TS, Dehlendorff C, et al. Hemorrhagic and ischemic strokes compared: stroke severity, mortality, and risk factors. Stroke 2009;40:2068-72.

10. Das S, Roy S, Kaul S, et al. E-selectin gene (S128R) polymorphism in hemorrhagic stroke: comparison with ischemic stroke. Neurosci Lett 2014;581:125-8.

11. Gryglas A, Smigiel R. Migraine and Stroke: What's the Link? What to Do? Curr Neurol Neurosci Rep 2017;17:22.

12. Lee MJ, Lee C, Chung CS. The Migraine-stroke connection. J Stroke 2016;18:146-56.

13. Eikermann-Haerter K, Lee JH, Yuzawa I, et al. Migraine mutations increase stroke vulnerability by facilitating ischemic depolarizations. Circulation 2012;125:335-45.

14. Timm FP, Houle TT, Grabitz SD, et al. Migraine and risk of perioperative ischemic stroke and hospital readmission: hospital based registry study. BMJ 2017;356:i6635.

15. Kurth T, Schürks M, Logroscino G, et al. Migraine, vascular risk, and cardiovascular events in women: prospective cohort study. BMJ 2008;337:a636.

16. Kurth T, Gaziano JM, Cook NR, et al. Migraine and risk of cardiovascular disease in women. JAMA 2006;296:283-91.

17. Monteith TS, Gardener H, Rundek T, et al. Migraine and risk of stroke in older adults: Northern Manhattan Study. Neurology 2015;85:715-21.

18. Spector JT, Kahn SR, Jones MR, et al. Migraine headache and ischemic stroke risk: an updated meta-analysis. Am J Med 2010;123:612-24.

19. Kuo CY, Yen MF, Chen LS, et al. Increased risk of hemorrhagic stroke in patients with migraine: a population-based cohort study. PLOS One 2013;8:e55253.

20. Sacco S, Ornello R, Ripa P, et al. Migraine and hemorrhagic stroke: a meta-analysis. Stroke 2013;44:3032-8.

21. Lee J, Lee JS, Park SH, et al. Cohort Profile: The National Health Insurance Service-National Sample Cohort (NHIS-NSC), South Korea. Int J Epidemiol 2017;46:dyv319.

22. NHISS. National health insurance data sharing service. http://nhiss. nhis.or.kr/

23. Kim RB, Kim BG, Kim YM, et al. Trends in the incidence of hospitalized acute myocardial infarction and stroke in Korea, 20062010. J Korean Med Sci 2013;28:16-24. 
24. Adelborg K, Szépligeti SK, Holland-Bill L, et al. Migraine and risk of cardiovascular diseases: danish population based matched cohort study. BMJ 2018;360:k96.

25. Mahmoud AN, Mentias A, Elgendy AY, et al. Migraine and the risk of cardiovascular and cerebrovascular events: a meta-analysis of 16 cohort studies including 1152407 subjects. BMJ Open 2018;8:e020498.

26. Tietjen GE. Migraine as a systemic vasculopathy. Cephalalgia 2009;29:989-96

27. Graif $Y$, Shohat $T$, Machluf $Y$, et al. Association between asthma and migraine: a cross-sectional study of over 110000 adolescents. Clin Respir J 2018;12:2491-6.

28. Papagianni M, Tziomalos K. Non-alcoholic fatty liver disease: an emerging predictor of stroke risk, severity and outcome. Eur J Neurol 2018;25:610-1.

29. Zhang Y, Parikh A, Qian S. Migraine and stroke. Stroke Vasc Neurol 2017;2:160-7.

30. Vuralli $\mathrm{D}$, Ayata $\mathrm{C}$, Bolay $\mathrm{H}$. Cognitive dysfunction and migraine. J Headache Pain 2018;19:109.

31. Tietjen EG. Migraine and ischaemic heart disease and stroke: potential mechanisms and treatment implications. Cephalalgia 2007;27:981-7.

32. Dreier JP, Major S, Manning A, et al. Cortical spreading ischaemia is a novel process involved in ischaemic damage in patients with aneurysmal subarachnoid haemorrhage. Brain 2009;132:1866-81.

33. Liman TG, Bachelier-Walenta K, Neeb L, et al. Circulating endothelia microparticles in female migraineurs with aura. Cephalalgia 2015;35:88-94.

34. Rajendran $P$, Rengarajan $\mathrm{T}$, Thangavel J, et al. The vascular endothelium and human diseases. Int J Biol Sci 2013;9:1057-69.

35. Pezzini A, Grassi M, Lodigiani C, et al. Predictors of migraine subtypes in young adults with ischemic stroke: the italian project on stroke in young adults. Stroke 2011;42:17-21.

36. De Giuli V, Grassi M, Lodigiani C, et al. Association between migraine and cervical artery dissection: the Italian project on stroke in young adults. JAMA Neurol 2017;74:512-8.
37. Malik R, Freilinger T, Winsvold BS, et al. Shared genetic basis for migraine and ischemic stroke: a genome-wide analysis of common variants. Neurology 2015;84:2132-45

38. Gaist D, González-Pérez A, Ashina M, et al. Migraine and risk of hemorrhagic stroke: a study based on data from general practice. $J$ Headache Pain 2014;15:74

39. Vlak MH, Rinkel GJ, Greebe P, et al. Risk of rupture of an intracranial aneurysm based on patient characteristics: a case-control study. Stroke 2013;44:1256-9.

40. Sacco S, Merki-Feld GS, Ægidius KL, et al. Hormonal contraceptives and risk of ischemic stroke in women with migraine: a consensus statement from the European Headache Federation (EHF) and the European Society of Contraception and Reproductive Health (ESC). J Headache Pain 2017;18:108.

41. Tsai CF, Anderson N, Thomas B, et al. Comparing risk factor profiles between intracerebral hemorrhage and ischemic stroke in Chinese and white populations: systematic review and meta-analysis. PLoS One 2016;11:e0151743.

42. Choi HG, Park B, Sim S, et al. Tonsillectomy does not reduce upper respiratory infections: a national cohort study. PLoS One 2016;11:e0169264.

43. Kim SY, Kim HJ, Park EK, et al. Severe hearing impairment and risk of depression: a national cohort study. PLoS One 2017;12:e0179973.

44. Kim MS, Kim SY, Kim JH, et al. Depression in breast cancer patients who have undergone mastectomy: a national cohort study. PLoS One 2017;12:e0175395.

45. Petty GW, Brown RD, Whisnant JP, et al. Ischemic stroke subtypes : a population-based study of functional outcome, survival, and recurrence. Stroke 2000;31:1062-8.

46. Bousser MG, Welch KM. Relation between migraine and stroke. Lancet Neurol 2005;4:533-42.

47. Roh J, Kim J, Ahn Y. Epidemiological and clinical characteristics of migraine in Korea. Journal of the Korean Neurological Association 1997;15:1-18. 